\title{
Prevalence of Non-Alcoholic Fatty Liver Disease (NAFLD) Diagnosed by Controlled Attenuation Parameter on Transient Elastography and Its Predisposing Factors in Psoriasis
}

\author{
Moonyza Akmal Ahmad Kamil ${ }^{1, *}$, Norazirah Md Nor ${ }^{2}$, Chai Soon Ngiu ${ }^{3}$, Mohd Rizal Abdul Manaf ${ }^{4}$, \\ Suganthi Thevarajah ${ }^{1}$, Adawiyah Jamil ${ }^{2}$ and Mohan Arumugam ${ }^{2}$ \\ ${ }^{1}$ Department of Dermatology, Hospital Kuala Lumpur, Kuala Lumpur, Malaysia \\ ${ }^{2}$ Dermatology Unit, Department of Medicine, Universiti Kebangsaan Malaysia (UKM) Medical Centre, Kuala Lumpur, Malaysia \\ ${ }^{3}$ Gastroenterology Unit, Department of Medicine, Universiti Kebangsaan Malaysia (UKM) Medical Centre, Kuala Lumpur, Malaysia \\ ${ }^{4}$ Department of Community Health, Universiti Kebangsaan Malaysia (UKM) Medical Centre, Kuala Lumpur, Malaysia \\ "Corresponding author: Department of Dermatology, Hospital Kuala Lumpur, Kuala Lumpur, Malaysia. Email: moonyza@yahoo.com
}

Received 2020 April 02; Revised 2020 April 14; Accepted 2020 April 18.

\begin{abstract}
Background: Psoriasis patients have an increased risk for non-alcoholic fatty liver disease (NAFLD). Controlled attenuation parameter (CAP) on transient elastography is more sensitive than abdominal ultrasonography in detecting liver steatosis.

objectives: To determine the prevalence of NAFLD in psoriasis and to identify its predisposing factors.

Methods: A cross-sectional study was performed involving 109 psoriasis patients aged $>18$ years in two tertiary dermatology clinics in Kuala Lumpur. Patients on hepatotoxic drugs, had excessive alcohol intake, and other secondary causes of chronic liver diseases were excluded. Anthropometrics, blood pressure, Psoriasis Area and Severity Index (PASI), liver function test, lipid profile, and fasting blood glucose were obtained. CAP on transient elastography was performed to diagnose NAFLD. The clinical characteristics of psoriasis patients were compared between patients with and without NAFLD.

Results: The prevalence of NAFLD was $85.3 \%$. NAFLD in psoriasis patients was associated with a higher weight $(\mathrm{P}<0.0005)$, body mass index (BMI) $(\mathrm{P}<0.0005)$, waist circumference $(\mathrm{P}<0.0005)$, and metabolic syndrome $(\mathrm{P}=0.002)$. Fasting blood glucose (5.3 $[1.8] \mathrm{mmol} / \mathrm{L}, \mathrm{p}=0.010)$, triglyceride $(1.4[0.8] \mathrm{mmol} / \mathrm{L}, \mathrm{P}<0.0005)$, and alanine transaminase (28.5 [26] U/L, $\mathrm{P}=0.001)$ were higher in patients with NAFLD compared with those without it. PASI and systolic blood pressure correlated significantly with NAFLD severity. $\mathrm{BMI}(\mathrm{OR}=1.63,95 \% \mathrm{CI}: 1.127-2.357, \mathrm{P}=0.009)$, triglyceride level $(\mathrm{OR}=130.74,95 \% \mathrm{CI}=2.94-812, \mathrm{P}=0.012)$, and $\mathrm{PASI}(\mathrm{OR}=1.138,95 \% \mathrm{CI}$ $=1.004-1.290, \mathrm{P}=0.043)$ were the significant predictors of NAFLD.

Conclusions: NAFLD should be screened in all psoriasis patients, especially in patients with high BMI, metabolic syndrome, and severe psoriasis, despite having a normal biochemical profile. Early detection of asymptomatic NAFLD is essential for preventive management, including to reduce hepatotoxicity risk of psoriasis pharmacotherapy.
\end{abstract}

Keywords: Psoriasis, NAFLD, Transient Elastography, Fibroscan, Non Alcoholic Fatty Liver Disease, Controlled Attenuation Parameter

\section{Background}

Psoriasis is a chronic, multifactorial immunemediated hyperproliferative inflammatory skin condition (1). It is relatively common with a prevalence rate of $1 \%$ - 3\% of the world population accounting for $2 \%-6 \%$ of yearly dermatology new clinic attendees in Malaysia $(2,3)$. Psoriasis is beyond skin deep and had been linked to other co-morbidities, including metabolic syndrome (MetS), obesity, and non-alcoholic fatty liver disease (NAFLD).

NAFLD is a spectrum of liver diseases, ranging from hepatosteatosis, which is harmless and characterized by non-inflammatory intracellular fat deposition, to nonalcoholic steatohepatitis (NASH), which occurs when fatty infiltration is accompanied by inflammation, with evidence of cellular injury and fibrosis (4). NAFLD may progress to cirrhosis and is a possible cause of cryptogenic cirrhosis (5). NAFLD is seen worldwide with a prevalence of $6 \%-45 \%$, which is closely related to MetS, type 2 diabetes mellitus (T2DM), dyslipidemia, and central obesity (6). In Malaysia, the prevalence of NAFLD in the suburban population was $22.7 \%$, and in patients with T2DM, the prevalence of NAFLD was almost $50 \%(7,8)$.

Although the pathogeneses of both entities are not

Copyright (c) 2020, Journal of Skin and Stem Cell. This is an open-access article distributed under the terms of the Creative Commons Attribution-NonCommercial 4.0 International License (http://creativecommons.org/licenses/by-nc/4.0/) which permits copy and redistribute the material just in noncommercial usages, provided the original work is properly cited. 
completely clear, it is thought that the increased levels of pro-inflammatory adipokines (e.g., TNF- $\alpha$ and IL-6), and hepatokines, with the presence of decreased levels of antiinflammatory adipokines (e.g., adiponectin) play a possible role in the chronic, low grade inflammation in both conditions. These imbalances could result in insulin resistance and facilitate the progression of NAFLD in psoriasis patients (9).

FibroScan is a clinically validated device using transient elastography indicated for the non-invasive measurement of liver stiffness (E) and controlled attenuation parameter (CAP), which allows the assessment and quantification of liver steatosis. It has a good correlation with the severity of steatosis and measures the liver volume up to 100 times larger than a liver biopsy. CAP measurement is not affected by liver fibrosis $(10,11)$. Although hepatobiliary ultrasound is the more common method for the screening of NAFLD, in the setting of mild steatosis, acuity decreases dramatically. Ultrasound also has substantial inter- and intra-observer variability (12). While transient elastography is operator independent, it provides reliable and reproducible quantitative measurements, and results are immediately available. With these advantages, transient elastography by using FibroScan was chosen as the mode of detecting NAFLD in our study. Detection of NAFLD in psoriasis patients will have clinical implications in preventive and treatment strategies.

\section{Objectives}

This study was performed to determine the prevalence of NAFLD in patients with psoriasis attending tertiary dermatology outpatient clinics in Kuala Lumpur, Malaysia, using CAP in transient elastography and to identify its predisposing factors.

\section{Methods}

An observational cross-sectional study was conducted prospectively on consecutive psoriasis patients aged $>18$ years in two dermatology tertiary centers in Kuala Lumpur (Hospital Kuala Lumpur and UKM Medical Centre) from November 2015 to May 2016. We excluded patients who had underlying secondary causes of chronic liver disease, patients taking traditional supplements, and patients currently or for the past three months on treatment with hepatotoxic medication. Patients who had excessive alcohol intake ( $>21$ units of alcohol per week for males and $>14$ units of alcohol per week for female patients) and those with contraindication for Fibroscan examination (e.g., pregnant, ascites) were also excluded.
A comprehensive history and physical examination, including demographic data, smoking and drinking habits, height, weight, blood pressure (BP), and waist circumference (WC), were obtained. In this study, metabolic syndrome is defined using NCEP-ATP III 2005 criteria, revised for Asians, which includes the presence of at least three risk factors (WC of $\geq 90 \mathrm{~cm}$ for males, $\geq 80 \mathrm{~cm}$ for females, systolic $\mathrm{BP} \geq 130$ and/or diastolic $\mathrm{BP} \geq 85$ or on treatment for hypertension, FBG $\geq 5.6 \mathrm{mmol} / \mathrm{L}$ or on treatment for elevated glucose). BMI classification used is based on the classification for adult Asians, as stated in the Clinical Practice Guidelines on Management of Obesity 2004, Ministry of Health, Malaysia.

The severity of psoriasis was assessed using the Psoriasis Area and Severity Index (PASI) by a single assessor. Patients with psoriatic arthritis (PsA) were classified according to Moll and Wright criteria.

Blood sample for full blood count consisting of hemoglobin, total white cell count, and platelet level, liver function test (LFT), comprised of total protein, albumin, bilirubin, alanine transaminase (ALT), alkaline phosphatase (ALP) and aspartate transaminase (AST); fasting serum lipids (FSL), comprising of total cholesterol, LDL-cholesterol, HDL-cholesterol and Triglyceride (TG), and fasting blood glucose (FBG) were sent for analysis at enrolment. All the participants were also screened for viral hepatitis by measuring hepatitis B surface antigen (HBsAg) and hepatitis C antibody (anti-HCV) with the enzyme immunoassay.

Transient elastography procedure was performed in the Gastroenterology Unit at UKM Medical Centre by a single experienced consultant gastroenterologist. For the purpose of this study, NAFLD was diagnosed using the CAP, which is available in the transient elastography using FibroScan 502. CAP is computed from ultrasounds acquired for liver stiffness measurement and is expressed in decibel per meter $(\mathrm{dB} / \mathrm{m})$. It is calculated only if liver stiffness measurement is valid. CAP is correlated with the grade of steatosis, with a cutoff value of $<215 \mathrm{~dB} / \mathrm{m}$ in the healthy population, $215-300 \mathrm{~dB} / \mathrm{m}$ in mild steatosis and $>300$ $\mathrm{dB} / \mathrm{m}$ in moderate to severe steatosis (13).

\subsection{Statistical Analysis}

Analysis of the research data was performed using SPSS version 23.0. The majority of our continuous variables were not normally distributed; thus, non-parametric tests were used for further data analysis. The tests used were chisquare, Fisher's exact test, Mann-Whitney U test, and Spearman rank order correlation. Multivariate analysis (logistic regression) was used to analyze the predictors of NAFLD. Data were analyzed in totality and further subdivided ac- 
cording to the presence or absence of NAFLD. A 2-tailed Pvalue of $<0.05$ is considered statistically significant.

\section{Results}

Of the 152 psoriasis patients screened during this sixmonth study, 33 patients were excluded initially, mainly due to the current usage of hepatotoxic drugs. Two patients had excessive alcohol intake, two patients had positive hepatitis B surface antigen on the screening test, and another patient was in her second trimester of her pregnancy. Twelve patients withdrew from the study because they were unable to comply with the study requirements. Therefore, 109 patients with psoriasis were finally included in data analysis.

In this study, the male to female ratio was 3:2. Patients were mostly overweight with a median (IQR) BMI of 25.73 $\mathrm{kg} / \mathrm{m}^{2}\left(4.86 \mathrm{~kg} / \mathrm{m}^{2}\right)$. Most of the patients had mild disease severity with a median(IQR) PASI score of 8.7(8.9). Only five patients had psoriatic arthropathy.

The prevalence of NAFLD in this study was $85.3 \%$. Out of this, 40 patients had mild NAFLD, while another 53 patients had moderate to severe NALFD.

In Table 1, psoriasis patients with NAFLD had significantly higher weight, BMI, and waist circumference, when compared to those patients without NAFLD. They were comparable in terms of age, ethnicity, gender, height, cigarette smoking and alcohol intake. The two groups were comparable with respect to age at the onset of psoriasis, duration of psoriasis, severity of psoriasis, presence of PsA, hypertension, diabetes and dyslipidemia. There was a significant association between MetS and NAFLD.

Psoriasis patients with NAFLD had significantly higher values of ALT than their counterparts without NAFLD, although they were still within the normal reference range. The levels of FBG and triglycerides were significantly higher, while the HDL-cholesterol level was significantly lower in the group of patients with NAFLD. The other biochemical parameters did not differ between the groups, as seen in Table 2.

There was a significant positive correlation between the severity of NAFLD and weight, BMI and waist circumference as depicted in Table 3. A weak positive correlation was also observed between the severity of NAFLD and the severity of psoriasis (measured by PASI) as well as the level of systolic blood pressure.

Factors that have significant correlation in the bivariate analysis were further analyzed using multivariate logistic regression analysis. The factors that were related to psoriasis include BMI, MetS, FBG, HDL-cholesterol, TG, ALT and SBP. Weight and waist circumference were not included as these variables are correlated with BMI.

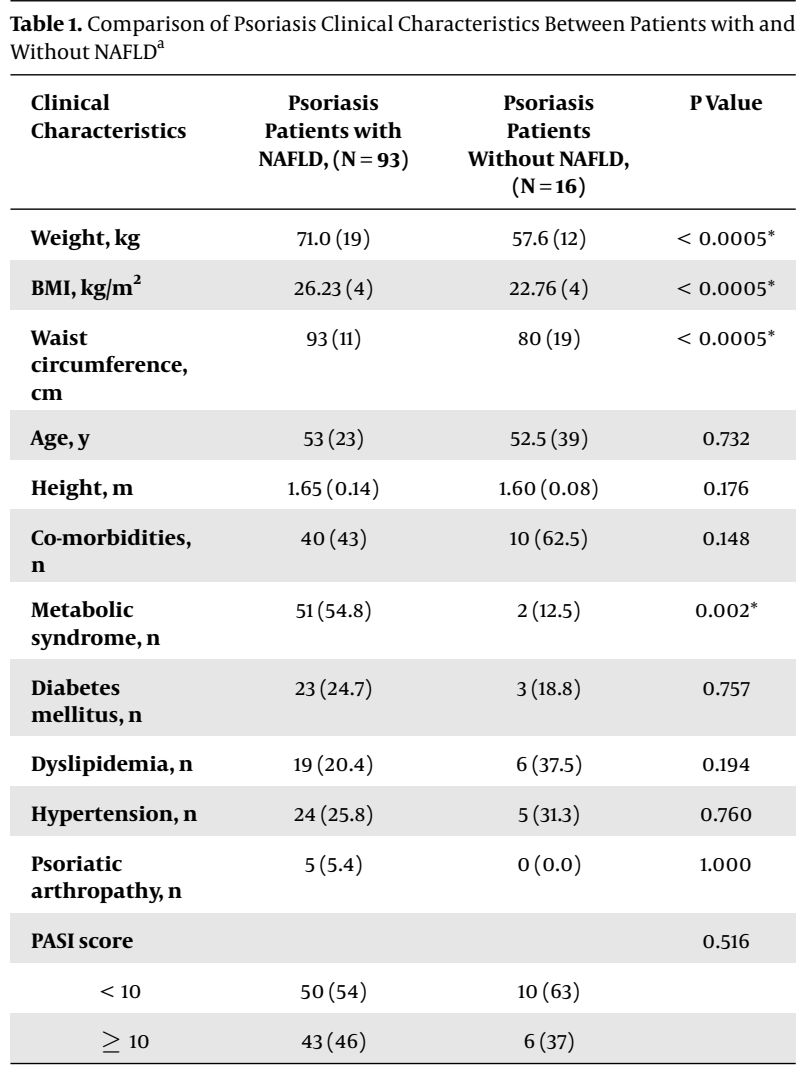

${ }^{\mathrm{a}}$ Values are expressed as No. (\%) or median (IQR).

Table 4 shows the variables that significantly predict the probability of having NAFLD. Nagelkerke R square is 0.601 indicates that $60.1 \%$ of the variance of having NAFLD is being explained by the significant factors. Among these three factors, BMI is the strongest predictor followed by TG and PASI (based on Wald values). Those who have one unit higher BMI, TG and PASI have 1.63,130 and 1.13 times higher odds to have NAFLD, respectively.

The chi-square test was performed to examine the relationship between the severity of steatosis and the presence of metabolic syndrome. The relationship between these variables was strongly significant, $\chi^{2}(2, \mathrm{n}=109)=34.81, \mathrm{P}$ $<0.001$. The chi-square test was also used to examine the association between the severity of psoriasis and the presence of metabolic syndrome. The relationship between these variables was also significant, $\chi^{2}(2, n=109)=6.65$, $\mathrm{P}=0.036$.

\section{Discussion}

To our knowledge, this is the first study to detect NAFLD using CAP on transient elastography in a cohort of psoriasis patients. We found a high prevalence of NAFLD in our 


\begin{tabular}{|c|c|c|c|}
\hline $\begin{array}{l}\text { Biochemical } \\
\text { Parameters }\end{array}$ & $\begin{array}{c}\text { Psoriasis } \\
\text { Patients with } \\
\text { NAFLD, }(\mathrm{N}=93)\end{array}$ & $\begin{array}{c}\text { Psoriasis } \\
\text { Patients Without } \\
\text { NAFLD },(\mathbf{N}=16)\end{array}$ & PValue \\
\hline FBG, $\mathrm{mmol} / \mathrm{L}$ & $5.30(1.8)$ & $4.90(0.4)$ & 0.010 \\
\hline $\begin{array}{l}\text { Total- } \\
\text { cholesterol, } \\
\text { mmol/L }\end{array}$ & $5.40(1.2)$ & $4.95(1.5)$ & 0.176 \\
\hline $\begin{array}{l}\text { LDL-cholesterol, } \\
\mathrm{mmol} / \mathrm{L}\end{array}$ & $3.49(1.3)$ & $3.0(1.1)$ & 0.058 \\
\hline $\begin{array}{l}\text { HDL-cholesterol, } \\
\mathrm{mmol} / \mathrm{L}\end{array}$ & $1.2(0.4)$ & $1.47(0.6)$ & 0.010 \\
\hline $\begin{array}{l}\text { Triglyceride, } \\
\text { mmol/L }\end{array}$ & $1.40(0.8)$ & $0.79(0.2)$ & $<0.0005$ \\
\hline Total protein, $\mathrm{g} / \mathrm{L}$ & $76(5)$ & $78(8)$ & 0.381 \\
\hline Albumin, $\mathrm{g} / \mathrm{L}$ & $42(6)$ & $45(6)$ & 0.890 \\
\hline $\begin{array}{l}\text { Bilirubin, } \\
\mu \mathrm{mol} / \mathrm{L}\end{array}$ & $11.65(6.4)$ & $12.40(4.8)$ & 0.653 \\
\hline ALT, U/L & $28.5(26)$ & $15.0(6)$ & 0.001 \\
\hline $\mathbf{A L P}, \mathbf{U} / \mathbf{L}$ & $79(21)$ & $75(20)$ & 0.722 \\
\hline AST, U/L & $24(13)$ & $20(3)$ & 0.62 \\
\hline $\begin{array}{l}\text { Hemoglobin, } \\
\text { g/dL }\end{array}$ & $14.25(2.6)$ & $13.2(2.0)$ & 0.172 \\
\hline Platelet, $\times 10^{9} / \mathrm{L}$ & $284.5(76)$ & $312(143)$ & 0.762 \\
\hline
\end{tabular}

Abbreviations: ALP, alkaline phosphatase; ALT, alanine transaminase; AST, aspartate aminotransferase; FBG, fasting blood glucose; FSL, fasting serum lipid. ${ }^{\mathrm{a}}$ Values are expressed as median (IQR)

Table 3. Correlation Between the Severity of Non-Alcoholic Fatty Liver Disease by the Measurement of CAP on Transient Elastography and Clinical Characteristics of Patients with Psoriasis ${ }^{\mathrm{a}}$

\begin{tabular}{lll}
\hline Clinical Characteristics & $\mathbf{r}_{\mathbf{s}}{ }^{\mathbf{b}}$ & PValue \\
\hline Age & -0.038 & 0.694 \\
\hline Weight & 0.574 & $<0.0005$ \\
\hline BMI & 0.642 & $<0.0005$ \\
\hline Waist circumference & 0.602 & $<0.0005$ \\
\hline PASI score & 0.202 & 0.035 \\
\hline Age at diagnosis & -0.021 & 0.829 \\
\hline Duration of psoriasis & 0.075 & 0.438 \\
\hline Systolic blood pressure & 0.191 & 0.047 \\
\hline Diastolic blood pressure & 0.146 & 0.130 \\
\hline $\begin{array}{l}\text { a Spearman's correlation. } \\
\text { b }{ }_{\mathrm{r}}=\text { Correlation coefficient. }\end{array}$ & & \\
\hline
\end{tabular}

Malaysian psoriasis patients (85.3\%). This figure is much higher compared to previous studies conducted among psoriasis patients in the western population, which ranges from $46.2 \%-59.2 \%$ (14-17). This prevalence rate is reported to be $4 \%, 17 \%$, and $65 \%$ in Taiwan (18), 17\% South India (19), and Iran (20), respectively.
The prevalence of NAFLD in the normal population in Malaysia was only $22 \%$ (8), thus, demonstrating that the prevalence of NAFLD among our psoriasis population was almost four folds higher than the normal Malaysian population.

One of the reasons for this higher prevalence of NAFLD seen in our study was maybe due to the higher sensitivity and specificity of transient elastography in detecting NAFLD compared to the conventional method with abdominal ultrasound scan. By comparison, most of the previous studies performed in North America and the European countries used abdominal ultrasound to detect NAFLD (14-17).

A much lower prevalence of fatty liver was defined by ICD-9-CM coding (code 571.8) (4\% of 51800 patients with psoriasis) in a study of psoriasis population performed by the National Health Insurance Database in Taiwan (18). However, a study in a predominantly rural population in South India detected NAFLD on the basis of abnormal liver enzymes in the presence of hepatic steatosis on ultrasonogram examination, amounting to a prevalence of only $17.4 \%$ out of 333 adult psoriasis patients (19).

The high prevalence of NAFLD in our cohort of patients could also be attributed to the mostly overweight and obese patients, with more than half meeting the criteria for MetS. Furthermore, this was in line with the Malaysian Psoriasis Registry in Malaysia, which reported $>50 \%$ of adult psoriasis patients were overweight and obese (21).

In our study, we found that NAFLD in psoriasis patients was associated with higher weight, BMI, waist circumference, and MetS. These findings were expected and comparable with previous studies, although different classifications were used for the definition of BMI and MetS in different cohorts of patients from various regions of the world $(14,19,22)$.

We did not find any significant difference in the severity of psoriasis between patients with NAFLD and those without NAFLD. This contradicts the finding by Gisondi et al. (15) where the frequency of NAFLD was remarkably greater in patients with severe psoriasis (PASI $\geq 10$ ). This difference could be explained by the fact that in our study, the majority of patients had mild to moderate psoriasis, while in that Italian study $>50 \%$ were in the severe spectrum. In our study, patients with more severe disease on hepatotoxic drugs such as methotrexate and acitretin were excluded. Nevertheless, we did observe a weak positive correlation between the severity of psoriasis and severity of NAFLD.

Notably, there was no significant difference in the presence of psoriatic arthropathy (PsA), which may be due to our small number of patients with PsA, as most of them were being treated with systemic agents and were ex- 


\begin{tabular}{|c|c|c|c|c|}
\hline Predictors & $\beta$ & Wald & PValue & OR $(95 \% \mathrm{CI})$ \\
\hline BMI & 0.489 & 6.736 & 0.009 & $1.630(1.127-2.357)$ \\
\hline Triglyceride & 4.873 & 6.336 & 0.012 & $130.737(2.94-5812.75)$ \\
\hline PASI & 0.129 & 4.087 & 0.043 & $1.138(1.004-1.290)$ \\
\hline HDL-cholesterol & 2.370 & 2.405 & 0.121 & $10.693(0.535-213.738)$ \\
\hline FBG & 1.084 & 1.421 & 0.233 & $2.958(0.497-17.595)$ \\
\hline ALT & 0.18 & 0.245 & 0.620 & $1.018(0.947-1.095)$ \\
\hline Systolic Blood Pressure & -0.013 & 0.251 & 0.616 & $0.987(0.937-1.039)$ \\
\hline
\end{tabular}

Abbreviations: ALT, alanine transaminase; BMI, body mass index; FBG, fasting blood glucose; PASI, Psoriasis area severity index.

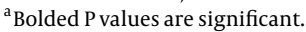

cluded. A systematic review and meta-analysis on the risk of NAFLD in patients with psoriasis showed that the risk of NAFLD was significantly greater in patients with psoriatic arthritis (three studies; $\mathrm{n}=505$ patients; $\mathrm{OR}=2.25,95 \% \mathrm{CI}=$ 1.37-3.71) and in patients with moderate to severe psoriasis compared to those with mild psoriasis (two studies; 51930 patients, $\mathrm{OR}=2.07,95 \% \mathrm{CI}=1.59-2.71)(22)$.

In terms of biochemical markers, those related to MetS were of significant difference in these two groups. In the group of psoriasis patients with NAFLD, the serum level of FBG and triglycerides were significantly higher, while the HDL-cholesterol level was significantly lower. This could be explained by the pathogenesis behind the metabolic syndrome. Insulin resistance and hyperinsulinemia increase the excretion of triglycerides by the liver, resulting in the elevated serum levels of triglycerides $(23,24)$. These findings correspond to the significantly higher percentage of MetS in the psoriasis group with NAFLD in comparison with the group without $\operatorname{NAFLD}(14,15,19)$.

The serum ALT level was significantly higher in the group with NAFLD, although most patients (88\%) had levels within the normal reference range. The degree of aminotransferase elevation does not predict the degree of hepatic inflammation or fibrosis, and a normal alanine aminotransferase does not exclude clinically important hepatocyte injury $(25,26)$. Therefore, the presence of NAFLD with normal liver enzymes should be taken into consideration when choosing the appropriate treatment, which is potentially hepatotoxic.

\subsection{Conclusions}

A high prevalence of NAFLD (85.3\%) was detected in psoriasis patients on transient elastography, despite normal biochemical profile. It is important to screen for NAFLD in all psoriasis patients, especially those with high BMI, MetS and severe psoriasis. Early detection of asymptomatic
NAFLD is essential to initiate appropriate management and prevent future complications of NAFLD.

\section{Acknowledgments}

The authors would like to thank the Director General of Health Malaysia for his permission to publish this article.

\section{Footnotes}

Authors' Contribution: Study concept and design: MAAK, NM, AJ, and ST. Acquisition of data: MAAK, and CN. Analysis and interpretation of data: MAAK, CN, MRAM, and NM. Drafting of the manuscript: MAAK. Critical revision of the manuscript for important intellectual content: MAAK, NM, AJ, and MRAM. Statistical analysis: MAAK, MRAM, and MA. Administrative, technical, and material support: MAAK, NM, AJ, and ST. Study supervision: NM, and AJ.

Conflict of Interests: None declared.

Ethical Approval: Ethical approval was obtained from the Medical Research and Ethics Committee (MREC) of Universiti Kebangsaan Malaysia (research code: FF-2015-346) and the approval from Malaysian National Medical Research Register (NMRR), Protocol number: NMRR 15-2006-27046.

Funding/Support: This work was supported by the UKMMC fundamental research fund of the Medical Research and Ethics Committee (MREC) of Universiti Kebangsaan Malaysia.

Informed Consent: Informed consent was signed by the participants. 


\section{References}

1. Griffiths CE, Barker JN. Pathogenesis and clinical features of psoriasis. Lancet. 2007;370(9583):263-71. doi: 10.1016/S0140-6736(07)61128-3. [PubMed: 17658397].

2. Raychaudhuri SP, Farber EM. The prevalence of psoriasis in the world. J Eur Acad Dermatol Venereol. 2001;15(1):16-7. doi: 10.1046/j.14683083.2001.00192.x. [PubMed: 11451313].

3. Siow KY, Safdar NA, Chong KH, Chua KB. A clinical appraisal of patients with psoriasis treated in Seremban General Hospital, Malaysia. Med J Malaysia. 2004;59(3):330-4. [PubMed:15727378].

4. Matteoni CA, Younossi ZM, Gramlich T, Boparai N, Liu YC, McCullough AJ. Nonalcoholic fatty liver disease: A spectrum of clinical and pathological severity. Gastroenterology. 1999;116(6):1413-9. doi: 10.1016/s0016-5085(99)70506-8. [PubMed:10348825].

5. Caldwell SH, Crespo DM. The spectrum expanded: cryptogenic cirrhosis and the natural history of non-alcoholic fatty liver disease. $J$ Hepatol. 2004;40(4):578-84. doi: 10.1016/j.jhep.2004.02.013. [PubMed: 15030972].

6. Younossi ZM, Stepanova M, Afendy M, Fang Y, Younossi Y, Mir H, et al. Changes in the prevalence of the most common causes of chronic liver diseases in the United States from 1988 to 2008. Clin Gastroenterol Hepatol. 2011;9(6):524-530 e1. quiz e60. doi: 10.1016/j.cgh.2011.03.020. [PubMed: 21440669].

7. Goh SC, Ho EL, Goh KL. Prevalence and risk factors of non-alcoholic fatty liver disease in a multiracial suburban Asian population in Malaysia. Hepatol Int. 2013;7(2):548-54. doi: 10.1007/s12072-012-9359-2. [PubMed: 26201786].

8. Chan WK, Tan AT, Vethakkan SR, Tah PC, Vijayananthan A, Goh KL. Nonalcoholic fatty liver disease in diabetics-prevalence and predictive factors in a multiracial hospital clinic population in Malaysia. J Gastroenterol Hepatol. 2013;28(8):1375-83. doi: 10.1111/jgh.12204. [PubMed: 23517307].

9. Prussick RB, Miele L. Nonalcoholic fatty liver disease in patients with psoriasis: A consequence of systemic inflammatory burden? Br J Dermatol. 2018;179(1):16-29. doi: 10.1111/bjd.16239. [PubMed: 29235656]

10. Sasso M, Tengher-Barna I, Ziol M, Miette V, Fournier C, Sandrin L, et al. Novel controlled attenuation parameter for noninvasive assessment of steatosis using Fibroscan $((\mathrm{R}))$ :Validation in chronic hepatitis C. J Viral Hepat. 2012;19(4):244-53. doi: 10.1111/j.1365-2893.2011.01534.x. [PubMed: 22404722].

11. Boursier J, de Ledinghen V, Sturm N, Amrani L, Bacq Y, Sandrini J, et al. Precise evaluation of liver histology by computerized morphometry shows that steatosis influences liver stiffness measured by transient elastography in chronic hepatitis C. J Gastroenterol. 2014;49(3):527-37. doi: 10.1007/s00535-013-0819-9. [PubMed: 23681425].

12. Strauss S, Gavish E, Gottlieb P, Katsnelson L. Interobserver and intraobserver variability in the sonographic assessment of fatty liver. AJR Am J Roentgenol. 2007;189(6):W320-3. doi: 10.2214/AJR.07.2123. [PubMed: 18029843].

13. Karlas T, Petroff D, Garnov N, Bohm S, Tenckhoff H, Wittekind C, et al. Non-invasive assessment of hepatic steatosis in patients with NAFLD using controlled attenuation parameter and 1H-MR spectroscopy. PLoS One. 2014;9(3). e91987. doi: 10.1371/journal.pone.0091987. [PubMed: 24637477]. [PubMed Central: PMC3956815].
14. Miele L, Vallone S, Cefalo C, La Torre G, Di Stasi C, Vecchio FM, et al. Prevalence, characteristics and severity of non-alcoholic fatty liver disease in patients with chronic plaque psoriasis. $J$ Hep atol. 2009;51(4):778-86. doi: 10.1016/j.jhep.2009.06.008. [PubMed: 19664838].

15. Gisondi P, Targher G, Zoppini G, Girolomoni G. Non-alcoholic fatty liver disease in patients with chronic plaque psoriasis. I Hepatol. 2009;51(4):758-64. doi: 10.1016/j.jhep.2009.04.020. [PubMed: 19560226].

16. Roberts KK, Cochet AE, Lamb PB, Brown PJ, Battafarano DF, Brunt EM, et al. The prevalence of NAFLD and NASH among patients with psoriasis in a tertiary care dermatology and rheumatology clinic. Aliment Pharmacol Ther. 2015;41(3):293-300. doi: 10.1111/apt.13042. [PubMed: 25521607].

17. van der Voort EA, Koehler EM, Dowlatshahi EA, Hofman A, Stricker BH, Janssen HL, et al. Psoriasis is independently associated with nonalcoholic fatty liver disease in patients 55 years old or older: Results from a population-based study. J Am Acad Dermatol. 2014;70(3):517-24. doi: 10.1016/j.jaad.2013.10.044. [PubMed: 24373781].

18. Tsai TF, Wang TS, Hung ST, Tsai PI, Schenkel B, Zhang M, et al Epidemiology and comorbidities of psoriasis patients in a national database in Taiwan. J Dermatol Sci. 2011;63(1):40-6. doi: 10.1016/j.jdermsci.2011.03.002. [PubMed: 21543188].

19. Madanagobalane S, Anandan S. The increased prevalence of nonalcoholic fatty liver disease in psoriatic patients: A study from South India. Australas J Dermatol. 2012;53(3):190-7. doi: 10.1111/j.1440 0960.2012.00905.x. [PubMed: 22672067].

20. Abedini R, Salehi M, Lajevardi V, Beygi S. Patients with psoriasis are at a higher risk of developing nonalcoholic fatty liver disease. Clin Exp Dermatol. 2015;40(7):722-7. doi: 10.1111/ced.12672. [PubMed: 25958919].

21. Mohd Affandi A, Ngah Saaya N, Johar A. Annual report of the Malaysian psoriasis registry 2007-2014. Malaysia; 2016.

22. Candia R, Ruiz A, Torres-Robles R, Chavez-Tapia N, Mendez-Sanchez $\mathrm{N}$, Arrese M. Risk of non-alcoholic fatty liver disease in patients with psoriasis: A systematic review and meta-analysis. J Eur Acad Dermatol Venereol. 2015;29(4):656-62. doi: 10.1111/jdv.12847. [PubMed: 25418531].

23. Parekh S, Anania FA. Abnormal lipid and glucose metabolism in obesity: Implications for nonalcoholic fatty liver disease. Gastroenterology. 2007;132(6):2191-207. doi: 10.1053/j.gastro.2007.03.055. [PubMed: 17498512].

24. Sozio MS, Liangpunsakul S, Crabb D. The role of lipid metabolism in the pathogenesis of alcoholic and nonalcoholic hepatic steatosis. Semin Liver Dis. 2010;30(4):378-90. doi: 10.1055/s-0030-1267538. [PubMed: 20960377].

25. Mofrad P, Contos MJ, Haque M, Sargeant C, Fisher RA, Luketic VA, et al. Clinical and histologic spectrum of nonalcoholic fatty liver disease associated with normal ALT values. Hepatology. 2003;37(6):128692. doi: 10.1053/jhep.2003.50229. [PubMed: 12774006].

26. Fracanzani AL, Valenti L, Bugianesi E, Andreoletti M, Colli A, Vanni E, et al. Risk of severe liver disease in nonalcoholic fatty liver disease with normal aminotransferase levels: A role for insulin resistance and diabetes. Hepatology.2008;48(3):792-8. doi:10.1002/hep.22429. [PubMed: 18752331]. 